\title{
The role of information modeling and automated technologies in the design and construction of high-rise buildings
}

\author{
Vera Cherkina ${ }^{1,{ }^{*}}$, Elena Petrenko ${ }^{2}$, Maxim Kirichenko ${ }^{1}$, and Pavel Samarin ${ }^{1}$ \\ ${ }^{1}$ Moscow State University of Civil Engineering, Yaroslavskoe sh. 26, Moscow, Russia \\ ${ }^{2}$ Kuban state agrarian university named after I. T. Trubilin, 3500440, Krasnodar, 13 Kalnina str., \\ Russia
}

\begin{abstract}
The purpose of the work is to consider the role of information modeling and automated technologies in the design and construction of high - rise buildings. The introduction of digital information tools in the construction sector provides rich ground for the birth and growth of companies specializing in the application of technology in design and construction. While some of the technologies are new, many companies implement ideas proposed in building research several decades ago that were impractical without a reliable digital database of building information. High-rise building information modeling (BIM) itself dates back to a landmark 1975 paper; ideas for artificial intelligence and codechecking tools emerged in the mid-1980s; and construction robots have been working in research labs for decades. However, only during the last five years, venture capital began to actively look for a new company in the sector of construction technologies. After analyzing a number of innovations in the field of digital construction through their known past and their uncertain present, it was concluded that the prospects for the development of these innovations in the construction industry, especially in the construction of high-rise buildings, are quite high. The author came to the following conclusion: a review of three specific areas of construction technologies, representing design and planning, BIM-to-field and field-toBIM applications, shows that the widespread adoption of BIM environments in the construction industry is not sufficient to ensure the effective use of the information they contain, or to use the potential of AI in this context.
\end{abstract}

\section{Introduction}

Researchers of architecture, engineering and construction have dreamed of applying information technology, robotics and other new technologies in design and construction for a long time. Invariably, however, their conceptual ideas about what could be done, and therefore their forward-looking views on the future of construction, far exceeded the

\footnotetext{
${ }^{*}$ Corresponding author: nskolch1501@gmail.com
} 
practical, technical, commercial, cultural, and / or organizational constraints that had to be overcome in order to accomplish them.

In 1975, attempts were made to develop a computerized building design system (BDS) with all the functionality of what is now known as building information modeling (BIM). It took 25 years for basic BIM functions to reach the market [1].

Over the past five years, there has been a constant inflow of new innovative companies specializing in the application of various information technologies and automation technologies developed in other industries, in construction. These start-up companies are supported by venture capitalists, academic research, and programs of public and private incubators, together with which they form an ecosystem commonly referred to as "Building technologies" (repeating the name "High technologies" used for the information and automated technology industry). In the United States, the amount of venture capital invested annually in Construction Tech has grown from about \$250 million in 2013 to more than $\$ 1,000$ million in 2019. [2]

Most new companies owe their newfound practicality not only to improvements in their core technologies, but equally to the extensive building information available in BIM environments [3]. In this context, the BIM environment includes various BIM tools, platforms, servers, libraries, and working process within a project or across an organization.

\section{Materials and methods}

In this paper, the term BIM is used in the broadest sense to include all software applications that generate, manipulate, store, and deliver information about buildings, rather than limiting it to a narrow set of platforms for creating models.

The study of information about high-rise buildings is based on the analysis of both projects of construction products and plans for the construction process. BIM environments provide this information, and this is the foundation on which new Construction Tech applications are created and bring benefits.

\section{Results}

Today there are four types of Construction Tech applications:

1. Software tools for design and construction management, most of which operate within or in close connection with model development platforms.

2. Software and hardware systems for delivering information from the project to the field - we call this group BIM-to-field tools. This group includes tools that deliver information via users ' mobile devices, as well as tools that deliver information about products and processes directly to the workplace in the field.

3. Robotic applications for performing construction work on the site.

4. Hardware and Software systems for collecting information from the site and transmitting it to control functions-we call this group field-to-BIM tools. These Construction Tech solutions provide huge amounts of data, but this data itself is of no value - you need to compare the planned conditions with the actual conditions in order to get valuable information [4].

Naturally, some applications will be used more than once - an application that delivers information about the process to the user's mobile device may also request information, for example, to update the current status.

BIM is an integrating technology that provides an information framework that transcends organizational boundaries within projects [5]. In this way, BIM environments support innovation in construction technologies, narrowing the gap between innovative 
technologies that use information technology and traditionally poor and fragmented organizations involved in construction projects. However, BIM platforms, tools, and processes cannot yet support most technological innovations out of the box. Therefore, it is necessary to study and work through the key aspects of barriers to information processing and data processing for construction technologies, focusing on aspects of BIM and artificial intelligence (AI) environments that require basic research to support commercial development.

We explore trends in construction developments over the past half-century, with the examples of research and development for automated compliance testing, design layout, construction robotics, and automated project monitoring and control. The growth of construction technologies based on BIM, field monitoring, robotics, artificial intelligence and other technologies will also be of interest [6].

Following the history of research and development (R \& D) in three broad areas of technological innovation in construction, we will trace their path to the present day. The goal is not to extrapolate into the future, but to identify key research objectives for continuous development - to identify the main research in the field of R \& D. The areas selected for review represent three of the four application types listed in the introduction:

1) software tools for design and planning in BIM environments;

2) BIM-to-field tools;

3) tools for converting the field to BIM that began to use digital doubles for construction [7].

Automating design and compliance testing in building construction has been a goal of research and commercial development since ideas were expressed in Eastman's landmark 1975 paper envisioning BIM. In the absence of BIM, researchers proposed Autonomous expert systems, and later systems that used CAD drawings to represent buildings. The first ones, such as HI-RISE for preliminary design of high-rise structures, SPEX for determining the size of structural sections, and EIDOCC for designing reinforced concrete columns, used symbolic information research (AI) methods [8].

Typically, these were rule-based systems that sought to gain expertise, capture it in design software, and apply it to automation or design verification. The need to explicitly and completely introduce building designs for each analysis, the limitations on knowledge acquisition, and the computing capabilities made these systems impractical for commercial applications. The advent of 2D CAD did not significantly improve the situation, since the graphical representations of design in CAD are fundamentally different from the semantic object-oriented representations required for processing rules.

The initial optimism that design standards could be expressed as rules and applied to the evaluation of building designs proved unfounded, as experiments revealed problems related to the lexical and logical complexity of building regulations. Later, natural language processing (NLP) was applied to building design norms and regulations, which led to some progress, but not to commercial application [8].

With the advent and implementation of BIM, computer-aided design and code validation have become more practical. Commercial model verification systems have been developed with limited but valuable and viable features (examples include Solibri Model Checker, BIM Assure, and SMARTreview). Although they can use BIM models, they require users to normalize the model data before use, and the set of code sentences they can check is limited to sentences that can be expressed as sets of symbolic IF-THEN rules (normalization is a pre-processing task of the BIM model for symbolic code verification. Users manually add or edit objects, parameter values, and relationships to conform to the object naming and typing conventions required by the rule sets).

Carrying out construction work on site is time-consuming and error-prone. The task is to interpret the design information in the context of partially completed scenes and apply 
physical markings on the surface with the necessary accuracy. The most modern method is the layout of a robotic total station, in which the operator determines the location of the total station using known points on the scene, and then "shoots" a laser beam to determine the location of the marking points. This is followed by manually displaying more complete design information from points to other surfaces using chalk lines, laser projectors, and other tools [9].

The scale of the immediate effort and subsequent refinement in the event of an error prompted R \&D to create automated layout systems that deliver BIM information directly to the field. Three types were suggested:

a) Augmented reality (AR) systems, in which the image of the intended design is superimposed on the image of the site recorded using a camera. AR systems require special glasses or masks, a tablet computer, or other device on which images are projected. Users must then translate this information to the work surface. These systems are especially useful to detect hidden system objects for the finished surfaces for the tasks of operation and maintenance of buildings or structures.

b) Robotic marking systems in which the robot localizes and then moves around the work area, applying paint or other marking material directly to the surface. They are usually limited to environments where the floors are clean and transparent, for marking and movement, and where the amount of marking work is large enough to justify the installation costs.

C) Robotic systems that project BIM information directly onto the work surface. For example, researchers have developed a prototype in which images from a BIM model are projected directly onto the work surface. The device consisted of a laser rangefinder, a projector with an adjustable tilt angle, and a camera. The system localizes itself using LIDAR and a BIM model using simultaneous localization and mapping (SLAM) and projects images containing information onto the work surface. It calibrates parameters for correcting trapezoidal projection distortions using image analysis [10].

Thanks to the growing capabilities of laser scanning and imaging technologies, improved localization accuracy, and sophisticated projection tools, this area seems to open up opportunities for the rapid development of new commercially viable tools. The Lightyx system is a good example of a startup development path for Construction Tech, where innovators with experience from other industries apply their knowledge of advanced technologies to solve construction problems. It is also an example of an innovation that fully fits into current construction practice, automating an isolated operation [11].

The concept of computer-aided testing (APPC) was proposed as a way to provide managers with the real-time feedback needed to apply the "thermostat"management model. The idea was to close the control loop by reporting key performance indicators such as labor and equipment productivity by monitoring the movement of workers and materials in real time. The concept of computer-aided testing (APPC) was proposed as a way to provide managers with the real-time feedback needed to apply the "thermostat"management model. The idea was to close the control loop by reporting key performance indicators such as labor and equipment productivity by monitoring the movement of workers and materials in real time. This direction of research may have anticipated the new concept of "Building 4.0 " or digital counterparts for construction, but it faced technical and conceptual obstacles:

- from a technical point of view, there was no available platform for integrating the necessary information about the production process for comparison with monitoring data. The researchers developed sophisticated methods for extracting information from documents and images of construction sites, but they were not linked to any integrated data management system; 
- from a conceptual point of view, the thermostat model turned out to be unsuitable and inefficient for planning and managing production in construction, and over time it was replaced by methods based on planning and managing exhaust production [12].

The idea of automating on-site monitoring came from the observation that field engineers spent a lot of time collecting performance data. A variety of technologies have been proposed for data collection, including computer vision, GPS, laser scanning, radio frequency identification tags, and low-power Bluetooth. However, with the exception of systems for monitoring heavy earthmoving equipment, field automation in BIM has not been adopted in the construction market.

A common thread runs through all three examples of Construction Tech innovations were detailed in the previous section: reliance is on the availability of digital information about buildings. Looking back, we see that this is not just a matter of digitizing information, but of providing free access to information on BIM platforms with syntactic and semantic compatibility. The current lack of direct access hinders the use of AI. In this section, we look at the current state of BIM and AI in the construction industry (around 2020) with reference to the same three areas of Construction Tech application [13].

While the advent of BIM has made commercial code-checking applications viable, their core technology has not changed fundamentally from what was envisioned in the 1980s. All of them use symbolic AI methods, primarily logical inference of rules, which limits their scope to relatively simple prescriptive provisions. The problem created by the large number of applicable design and construction standards and the frequency of their updates has not been resolved. Commercial applications still require explicit representation of building information, and the effort required for normalization limits their use to individual stages in design processes [14].

Revolutionary progress in code verification will require overcoming these barriers and new approaches and technologies. Among the most promising:

- semantic enrichment of BIM models using artificial intelligence techniques to automatically supplement models with explicit information obtained using algorithms trained to recognize and output predefined sets of target concepts in construction data templates may offer a way to eliminate the need for normalization;

- applying machine learning algorithms to evaluate projects based on training data and results from human experts;

- graphical representations of BIM models can offer explicit necessary representations, in particular, in order to make explicit the relationships between building objects and abstract concepts. They are also more adapted to the types of pattern recognition algorithms that can provide semantic enrichment and training of machine learning algorithms [15].

It must be said that all companies offering commercial applications for checking BIM code are start-up companies (Compliance Audit Systems Limited, Daima, Invicara, SMARTreview) or started as start-up companies (Solibri, recently acquired by Nemetschek).

The delivery of BIM information to the site is largely resolved with regard to the delivery of BIM information to staff via mobile computing devices. All major companies involved in the BIM platform offer solutions, most of which were created by breakthrough Construction Tech start-UPS whose solutions were acquired by well-known BIM companies (for example, PlanGrid, Trimble Connect, Solibri). Tools that present information about the model using augmented reality are also available (for example, Trimble XR10 with Hololens 2; Trimble 2020 ), although they still suffer from practical problems such as a narrow field of view, blurry display in bright environments, difficulty working, etc. [16]

Despite ten or more years of industrial research and development, there are still no commercially viable solutions for placing directly on work surfaces. One of the key tasks is 
to project or label information on non - standard intermediate work surfaces in the real world, since they do not directly correspond to the ideal designed surfaces of finished products that exist in the virtual world of BIM models. Ironically, this problem could be overcome if "field-to-BIM" technologies were able to build accurate virtual digital dual representations of site conditions. However, as we will discuss below, this also remains a problem.

Automated monitoring and control of project execution is full with solutions offered by both recognized software and hardware vendors and start-up companies. Applications range from verification systems that allow inspectors to access data before, during, and after the verification process and access recording functions for collecting data on the site, to control systems that allow monitoring security, site attendance, resources, storage space usage, and others. for (iii) planning and measurement systems, logistics and location planning, production monitoring, etc. Some investments have been invested in this site; however, all applications are single-track and operate as information Islands. They use one or more data collection technologies and interpret them, as far as possible, into useful information for construction management (for example, Siteaware, Disperse.io, Holobuilder, Smartvid.io, Versatile Natures, Openspace.ai, Genda and others). The information they provide is not always reliable and requires manual analysis and intervention, which often negates their automation advantages. Their limited approach also limits what conclusions can be drawn [17].

In fact, there is a need for complex event processing. This will involve combining information from multiple controlled data sources with pre-existing information about the current state of the construction site and production plan to output accurate information about what was built, how and what resources were used, and where to combine data from multiple sources to compile comprehensive information about the project status (in terms of both product and process status). However, complex event processing is only possible on the basis of well-integrated and reliable data, which we still lack. Despite the obvious abundance of data, the current lack of complete, accurate, and reliable related data and information naturally limits opportunities for proper use of technology [18].

\section{Discussion}

In theory, BIM models of buildings and infrastructure are ideal for manipulating intelligent software tools that include computer vision, rule definition, machine learning, case-based reasoning, and other AI strategies. The range of potential applications is wide, including, but not limited to, smart tools for:

- design support and / or automation, topology optimization, generative design;

- design review, verification of compliance with standards and regulations;

- building performance modeling and engineering analysis;

- construction planning, site layout development, supply chain management;

digital delivery of design and construction methods directly to on-site workers;

- real-time measurement, evaluation, and interpretation of project status;

-quality assurance and control;

- production control, resource allocation, material and information flow management;

- security planning and control;

- assessment of the cost of sustainability and life cycle;

- getting BIM models from point cloud data, photo and videogrammetry;

-operation and management of objects using digital doubles [19].

Computer science researchers in architecture, design, and construction (AEC) around the world have sought to implement such tools since the development of the ideas behind AI. In the early 1980s and 1990s, people tried to apply expert systems and case-based 
reasoning to solve some of the problems listed above. It soon became apparent that CAD technology was not suitable for such applications, because its representation of building information was graphical and symbolic, rather than object-oriented. This has led to intensive efforts to address the representation problem, leading to platforms for developing BIM models that are now ubiquitous in the industry, and an open object-oriented schema for representing buildings and infrastructure (the IFC data model).

It is obvious that the problem of presenting information has been solved, and a platform for commercial innovation has appeared, and a wave of technological innovations has begun. Most of the innovation funding was provided to startups. There are two main reasons for this: corporate / organizational fragmentation in the industry and the need for expertise and experience with technologies adapted from other industrial areas [20].

The literature provides strong evidence for innovations that develop outside of construction design organizations due to industry fragmentation: vertical fragmentation (professional and trade specialization), horizontal fragmentation (several small firms compete with each other), and longitudinal fragmentation (high turnover). and clients from project to project). In this environment, system innovations tend to break existing commercial or organizational boundaries and therefore require completely new vertically and longitudinally integrated organizations with high initial costs and significant risk.

In this context, it is not surprising that many innovators in the field of construction technologies cannot overcome regulatory, commercial, cultural, organizational and technological barriers, despite inventing and developing advanced technological applications in the field of construction. Taking into account these risks, almost all innovators take a step-by-step approach to changes in the construction industry, since their main priority is to create a minimum viable product and generate revenue [21].

In addition to the basics of entrepreneurship (ideas, investment and implementation), all innovators in the field of construction technologies need at least three important things:

1) real production needs in the industry;

2) applying a new technology that meets this need;

3) a workable business model. These are the primary elements of the "House of building technologies", which we offer as a model to explain the components necessary for success in this sector [22].

Entrepreneurship creates beams that support the roof, which is the top of success - the introduction of the construction industry in the market. The BIM environment, in the broadest sense, as technology, process, and people, is at the heart of the home. BIM technology is hardware and software that generates and stores information about a construction project, including its physical aspects (building design) and its technological aspects (construction plans). BIM processes are aspects of information management standards such as ISO 19650 and IFC); BIM implementation plans at the organization and project level; definition of the level of detail (LOD); and so on. [23].

People are initiators who are able to implement processes using technology, including not only employees of the innovator (designers, programmers, etc.), but, equally important, employees of customers (architects, engineers, construction managers) who have qualifications in working in a BIM environment.

Construction information in a form that can be easily manipulated by software is important for almost all innovations in construction technology and, therefore, for placing the BIM environment at the base of a house. All four types of applications (design and management, BIM-to-field, field automation, and field-to-BIM) depend on information in one form or another. The maturation of BIM environments and their widespread adoption is one key common denominator supporting the growth of construction technologies over the past decade. However, equally, BIM technology and processes still have serious limitations that are holding back the long-awaited growth and success of some Construction Tech 
applications. Among the main limitations are inadequate information compatibility, difficulties in generating model data for machine learning applications for design and management.[24]

While the prospects for innovation in construction technologies are promising, there are still challenges that need to be considered. Some of the technological limitations mentioned in the sections above, which describe the known past and the uncertain present, show that the presentation of information is still inadequate. Serious fundamental problems still need to be solved before Construction Tech innovators can begin to widely implement artificial intelligence methods in intelligent software tools that manipulate BIM models [25].

One of the key problems is that models are usually discipline-dependent, representing buildings with the semantics of a single professional representation (for example, architecture, structural design, or MEP systems). Thus, interdisciplinary collaboration using models is difficult, since most teams use federations of individual models. Another key problem is that many relationships and properties of objects are still implicit in BIM models, left to the discretion of their human users.

The third problem is that even where the information is complete, object-oriented representations are not suitable for representations of objects and their characteristics, which are standard input for existing AI methods. Similarly, design specifications and building codes usually define parameters that are a complex compilation of geometric and other constraints that are very difficult to Express using sets of "if-then" rules. Objectoriented representations are not suitable for representations of objects and their characteristics, which are the standard input of existing AI methods [26].

These issues are common to all aspects of developing tools for applications described above. Some problems can be solved through semantic enrichment, in which professional knowledge is used to infer missing information from the target point of view of intelligent tools that receive data from models. In general, this would make the information complete. Others require the development of data engineering strategies to make the information compatible for AI processing [27].

At the implementation level, we identify two separate research tasks for the development of basic information processing methods for BIM models, the solution of which would greatly simplify the development of a wide range of intelligent BIM and AI tools for design and construction:

1) combined optimal use of topological rule inference modules and machine learning for semantic enrichment;

2) representations of BIM models suitable for AI applications, with a focus on machine learning [28].

Semantic enrichment of BIM models is a process in which algorithms apply expert knowledge to output any information needed for a given application that is not present in the explicit data in the model. Models are incomplete representations of buildings. An explicit set of geometry, properties, and relationships in the model is insufficient input for most host applications from subdomains other than the one in which it was created. The output information can be added to the model, enriching it and making it easier to use for any given receiving application. Semantic enrichment includes classification of construction objects, aggregation and grouping of objects into functional groups or systems, unique identification of building elements, creation of missing objects, properties and relationships, and reconstruction of closed objects when applied to models compiled from the cloud of points.

Semantic enrichment is not a Mature technology. This is a relatively new area of research, and the literature on this topic is limited. Much work has been done on intelligent semantic query of BIM models, and these efforts have begun to use meaningful topological 
constructs of information that is implied in models but not explicitly stored. They use an implicit value, but no information is added to enrich the model during their operations.

Software modules for semantic enrichment is focused on the achievement of objectives. Missing or distorted information that needs to be supplemented is defined in the domainspecific model representation definition (MVD). The first experimental applications applied rule inference, a subset of artificial intelligence methods in which system logic is defined as a set of rules, usually in the form of "IF-THEN". In straight-chain solutions, rules are processed iteratively, adding new information to the model whenever the rule is evaluated as TRUE. The iteration terminates when it is impossible to derive new facts. A reverse chain is also possible, where rules are used to test hypotheses and search for evidence in existing information. More recent research has shown that some aspects of semantic enrichment can be better performed using machine learning [29].

Unsupervised machine learning has previously been applied to BIM models to detect anomalies and misclassified objects. Discrepancies in IFC-based data exchange are also resolved by using machine learning algorithms such as Support Vector Machine (SVM) to detect misclassification of specific objects in IFC files. In the same way, the authors applied learning algorithms to determine common construction objects in IFC files [30].

Fundamental research is needed to classify BIM information objects according to their suitability for semantic enrichment using various artificial intelligence methods (primarily topological rule inference, supervised machine learning, or deep learning), as well as to develop sets of software semantic enrichment methods that can be compiled to create applications in a modular fashion. If these goals can be achieved, the main problem of BIM compatibility can be solved, since standard models can be enriched for almost any purpose. This will provide a technological Foundation for Construction Tech startups to implement a wide range of applications (some of which are listed in the conclusions section below).

Building models are stored in one of three ways - in proprietary file formats specific for particular BIM development platforms, in open IFC files based on EXPRESS, or, more recently, in proprietary cloud databases. None of these formats are directly compatible with image recognition and / or machine learning algorithms. In all existing artificial intelligence applications that use BIM information, whether full models, partial assemblies from models, or model components, users must extract and compile the relevant information again for every use. Extraction usually requires exporting table schedules of objects and their properties, or analyzing IFC files.

The main problem with these methods is that significant information is lost during translation. Perhaps most significant is the loss of links between objects that are available in the native representation of the BIM software, but that cannot be easily exported in table schedules and are usually completely absent from IFC export files. Examples are the relationships of embedding between Windows and walls and the relationships of loadbearing structures between beams and columns. As a result, solutions either use narrow aspects of building object properties in isolation, or require extensive programming to extract and express building object relationships as characteristics of individual objects.

This is a serious limitation when you consider that the networks of relationships between construction objects form completely different patterns. For example, the rulebased methods developed for classifying bridge components in the SeeBridge project relied heavily on topological relationships between objects. In this case, the information was analyzed from IFC files, and the relationships were inferred by the rule processing system from the physical location of objects relative to each other before they could be classified.

The complexity of expressing model objects and their relationships also points to a promising solution - representing building models as property graphs. Researchers have developed a set of tools for compiling a graph data model from the building model IFC file. Their method evaluated the geometry of building objects to determine topological 
relationships and supplement the graph model with connections, coupling, placement, division, and intersection, which were modeled as weights on the edges of the graph [30].

Other authors used a more appropriate representation of the property graph, creating both the IFC schema graph from EXPRESS and the IFC model graphs for individual instances of building models stored in IFC files. They did not transform the geometry or derive topological relationships from the geometry sufficient to Express the information contained explicitly in IFC files. While none of these attempts at semantic enrichment using domain-specific knowledge beyond the five main topological relationships, the graphs presented and the use cases they demonstrated with these representations strongly support the idea that property graphs are suitable for building modeling for applications. which require machine learning and / or pattern matching [31].

\section{Conclusion}

A review of three specific areas of construction technologies, representing design and planning, BIM-to-field and field-to-BIM applications, shows that the widespread adoption of BIM environments in the construction industry is not sufficient to ensure the effective use of the information they contain, or to use the potential of AI in this context. The problem is that the information in the models is incomplete and unavailable. Among the many technological challenges faced by entrepreneurs in the field of construction technologies, there are two specific research tasks that relate to the development of fundamental information processing methods for digital information models of buildings, the solution of which will greatly simplify the development of intelligent BIM and AI tools for design and construction. They are:

1) combined optimal use of topological rule inference modules and machine learning for semantic enrichment;

2) encoding representations of construction information in forms that lend themselves to machine learning.

As for the nature of innovation in construction technology, our review of applications confirms researchers ' predictions that technological innovation in construction is more likely to come from disruptive startups than from traditional project-oriented construction companies. The growth in investment in Construction Tech startups shows that the market shares this view. The "House of Construction Tech" model can help both investors and innovators evaluate the reliability of their startups ' technologies and business strategies. It should be taken into account that the model applies to incremental innovation; a complete rethink of the construction business model may require a rethink of the underlying technologies as well.

Naturally, this paper does not cover all possible technological problems for implementing AI and BIM applications. There may be others, and presumably new problems will arise, even if solutions for semantic enrichment and graphical representation of BIM models are developed and implemented. However, these two aspects are the key to progress and, accordingly, deserve the attention of researchers.

\section{References}

1. M. Al Qady, A. Kandil, Concept Relation Extraction from Construction Documents Using Natural Language Processing, Journal of Construction Engineering and Management 136 (3), 294-302 (2010)

2. G. Ballard, The Lean Project Delivery System: An Update Lean Construction Journal 2008, 1-19 (2008) 
3. M. Belsky, R. Sacks, I. Brilakis Semantic enrichment for building information modeling Computer-Aided Civil and Infrastructure Engineering 31 (4), 261-274 (2016)

4. T. Bloch, R. Sacks, Comparing machine learning and rule-based inferencing for semantic enrichment of BIM models, Automation in Construction 91, 256-272 (2018)

5. I. Brilakis, L. Soibelman, Y. Shinagawa, Material-Based Construction Site Image Retrieval Journal of Computing in Civil Engineering 19(4), 341-355 (2005)

6. H.-L. Chi, S.-C. Kang, X. Wang, Research trends and opportunities of augmented reality applications in architecture, engineering, and construction, Automation in Construction, Augmented Reality in Architecture, Engineering, and Construction 33, 116-122 (2013)

7. T. Chowdhury, J. Adafin, S. Wilkinson, Review of digital technologies to improve productivity of New Zealand construction industry, Journal of Information Technology in Construction 24, 569-587 (2019)

8. A. Costin, N. Pradhananga, J. Teizer, Leveraging passive RFID technology for construction resource field mobility and status monitoring in a high-rise renovation project, Automation in Construction 24, 1-15 (2012)

9. A. Degani, W.B. Li, R. Sacks, L. Ma, An Automated System for Projection of Interior Construction Layouts IEEE Transactions on Automation Science and Engineering 16 (4), 1825-1835 (2019)

10. J. Dimyadi, P. Pauwels, R. Amor, Modelling and accessing regulatory knowledge for computer-assisted compliance audit ITcon, Special issue CIB W78 2015 Special track on Compliance Checking 21, 317-336 (2016)

11. P. Galle, Philosophy of design: an editorial introduction Design Studies 23 (3), 211218 (2002)

12. J.H. Garrett, S.J. Fenves, A knowledge-based standards processor for structural component design Engineering with Computers 2 (4), 219-238 (1987)

13. M. Hakim, J.H. Garrett, Using Description Logic for Representing Engineering Design Standards, Journal of Engineering with Computers 9, 108-124 (1993)

14. D.M. Hall, J.K. Whyte, J. Lessing, Mirror-breaking strategies to enable digital manufacturing in Silicon Valley construction firms: a comparative case study Construction Management and Economics (2019)

15. B. Koo, S. La, N.-W. Cho, Y. Yu, Using support vector machines to classify building elements for checking the semantic integrity of building information models, Automation in Construction 98, 183-194 (2019)

16. B. Koo, B. Shin, Applying novelty detection to identify model element to IFC class misclassifications on architectural and infrastructure Building Information Models, Journal of Computational Design and Engineering 5 (4), 391-400 (2018)

17. S. Lee, Ö. Akin, Augmented reality-based computational fieldwork support for equipment operations and maintenance, Automation in Construction 20 (4), 338-352 (2011)

18. W. Mazairac, J. Beetz, "BIMQL? An open query language for building information models, Advanced Engineering Informatics 27 (4), 444-456 (2013)

19. B.G. McCullouch, Automated Construction Field-Data Management System, Journal of Transportation Engineering 118 (4), 517-526 (1992)

20. B.G. McCullouch, K. Lueprasert, 2D Bar-Code Applications in Construction Journal of Construction Engineering and Management 120 (4), 739-752 (1994) 
21. R. Navon, Automated project performance control of construction projects, Automation in Construction 14 (4), 467-476 (2005)

22. T.-H. Nguyen, A.A. Oloufa, Computer-Generated Building Data: Topological Information, Journal of Computing in Civil Engineering 15 (4) (2001), pp. 268-274 (2001)

23. R. Sacks, What constitutes good production flow in construction? Construction Management \& Economics 34 (9), 641-656 (2016)

24. R. Sacks, O. Buyukozturk, Expert Interactive Design of Concrete Columns under Biaxial Bending ASCE, Journal of Computing in Civil Engineering 1, 69-81 (1987)

25. R. Sacks, C.M. Eastman, G. Lee, Process Improvements in Precast Concrete Construction Using Top-Down Parametric 3-D Computer-Modeling, Journal of the Precast/Prestressed Concrete Institute 48, 46-55 (2003)

26. R. Sacks, C.M. Eastman, G. Lee Parametric, 3D Modeling in Building Construction with Examples from Precast Concrete Automation in Construction 13, 291-312 (2004)

27. R. Sacks, R. Navon, I. Brodetskaia, Interpretation of Automatically Monitored Equipment Data in Construction Control, Journal of Computing in Civil Engineering 20, 111-120 (2006)

28. Z. Turk, T. Isakovic, M. Fischinger, Object-Oriented Modeling of Design System for $R C$ Buildings, Journal of Computing in Civil Engineering 8, 436-453 (1994)

29. X. Wang, M.J. Kim, P.E.D. Love, S.-C. Kang, Augmented Reality in built environment: Classification and implications for future research, Automation in Construction 32,1-13 (2013)

30. A. Warszawski, D.A. Sangrey, Robotics in Building Construction, Journal of Construction Engineering and Management 111 (3), 260-280 (1985)

31. J. Zhang, N.M. El-Gohary, Integrating semantic NLP and logic reasoning into a unified system for fully-automated code checking, Automation in Construction 73, 4557 (2017) 